\title{
Weight Function and Nested Bethe Ansatz
}

\author{
Stanislav Pakuliak* \\ Dedicated to the memory of Daniel Arnaudon
}

\begin{abstract}
The projections of the product of the Drinfeld's currents onto intersections of the different Borel subalgebras in the current realization of the quantum affine algebra $U_{q}\left(\widehat{\mathfrak{g l}}_{N}\right)$ are calculated. It was shown that this projections has the structure of the off-shell nested Bethe ansatz vectors.
\end{abstract}

\section{Introduction}

Off-shell Bethe vectors in the $\widehat{\mathfrak{g l}}_{N}$ integrable models appeared in [KR] in the framework of the hierarchical or nested Bethe ansatz. They were necessary for realization of the form-factor program in the integrable models associated with higher rank algebras and for description of the integral solutions to the quantized KnizhnikZamolodchikov equations [TV1, TV2]. Recently, a new approach for the construction of the off-shell Bethe vectors was proposed in the papers [KhP, EKhP]. This method can be formulated for any quantized current algebra and uses certain projections onto intersections of the different type of Borel subalgebras.

One of purposes of this paper is to compare the two constructions of the weight functions given in terms of $L$-operators [TV1] and in terms of projections [EKhP] for the quantum algebra $U_{q}\left(\widehat{\mathfrak{g l}}_{N}\right)$. A weight function is a vector-valued function taking values in $U_{q}\left(\widehat{\mathfrak{g l}}_{N}\right)$-module generated by a singular weight vector. We will prove coincidence of the two constructions for the symmetric tensor products of the fundamental vector representations of $U_{q}\left(\widehat{\mathfrak{g l}}_{N}\right)$, where calculations are easy and the weight functions in this case have relatively simple structure. We will demonstrate, that weight functions defined by the projections and obtained using $U_{q}\left(\widehat{\mathfrak{g l}}_{N}\right)$ monodromy operator satisfy the same recursion relations on the rank $N$. This proves their coincidence. We will extensively use the isomorphism between $L$-operator [RS] and current [D] formulation of $U_{q}\left(\widehat{\mathfrak{g l}}_{N}\right)$ established in [DF].

* Supported in part by the grants INTAS-OPEN-03-51-3350 and RFBR grant 05-01-086. 


\section{Off-shell Bethe vectors}

2.1. $L$-operator description of $U_{q}\left(\widehat{\mathfrak{g l}}_{N}\right)$

Let $E_{i j} \in \operatorname{End}\left(\mathbb{C}^{N}\right)$ be a matrix with the only nonzero entry equal to 1 at the intersection of the $i$-th row and $j$-th column. Matrix

$$
\begin{aligned}
R(u, v) & =\frac{q u-q^{-1} v}{u-v} \sum_{1 \leq i \leq N} E_{i i} \otimes E_{i i}+\sum_{1 \leq i<j \leq N}\left(E_{i i} \otimes E_{j j}+E_{j j} \otimes E_{i i}\right) \\
& +\frac{q-q^{-1}}{u-v} \sum_{1 \leq i<j \leq N}\left(u E_{i j} \otimes E_{j i}+v E_{j i} \otimes E_{i j}\right)
\end{aligned}
$$

is called $U_{q}\left(\widehat{\mathfrak{g l}}_{N}\right) R$-matrix. It depends actually on the ratio of the spectral parameters $R(u, v)=R(u / v)$ and should be understood as series over powers of the ratio $u / v$. It satisfies the Yang-Baxter equation

$$
R^{12}(u / v) R^{13}(u) R^{23}(v)=R^{23}(v) R^{13}(u) R^{12}(u / v)
$$

and the unitarity relation

$$
R^{(12)}\left(u_{1}, u_{2}\right) R^{(21)}\left(u_{2}, u_{1}\right)=\frac{\left(q u_{1}-q^{-1} u_{2}\right)\left(q^{-1} u_{1}-q u_{2}\right)}{\left(u_{1}-u_{2}\right)^{2}} .
$$

Let $U(R)$ be an associative algebra with unity generated by the elements $L_{i j}^{ \pm}[ \pm k]$, for $k>0,1 \leq i, j \leq N$ and $L_{j i}^{+}[0], L_{i j}^{-}[0]$ for $1 \leq i \leq j \leq N$. Define generating series $L^{ \pm}(z)=\sum_{k=0}^{\infty} L_{i j}^{ \pm}[ \pm k] z^{\mp k}$, where $L_{i j}^{+}[0]=L_{j i}^{-}[0]=0$ for $1 \leq$ $i<j \leq N$ and $L_{i i}^{+}[0] L_{i i}^{-}[0]=1$ for $1 \leq i \leq N$. Let us combine these generating series into $N \times N$ matrices $L^{ \pm}(z)=\sum_{i, j=1}^{N} E_{i j} \otimes L_{i j}^{ \pm}(z) \in \operatorname{End}\left(\mathbb{C}^{N}\right) \otimes U(R)$. These matrices are called $L$-operators. The commutation relations in the algebra $U(R)$ can be written as relations in $\operatorname{End}(\mathbb{C}) \otimes \operatorname{End}(\mathbb{C}) \otimes U(R)$

$$
\begin{aligned}
& R(u, v) \cdot\left(L^{ \pm}(u) \otimes \mathbf{1}\right) \cdot\left(\mathbf{1} \otimes L^{ \pm}(v)\right)=\left(\mathbf{1} \otimes L^{ \pm}(v)\right) \cdot\left(L^{ \pm}(u) \otimes \mathbf{1}\right) \cdot R(u, v), \\
& R(u, v) \cdot\left(L^{+}(u) \otimes \mathbf{1}\right) \cdot\left(\mathbf{1} \otimes L^{-}(v)\right)=\left(\mathbf{1} \otimes L^{-}(v)\right) \cdot\left(L^{+}(u) \otimes \mathbf{1}\right) \cdot R(u, v),
\end{aligned}
$$

where $\mathbf{1}=\sum_{i=1}^{N} E_{i i}$ is an identity matrix.

Coalgebraic structure of the algebra $U(R)$ is defined by the formulas

$$
\Delta\left(L_{i j}^{ \pm}(u)\right)=\sum_{k=1}^{N} L_{k j}^{ \pm}(u) \otimes L_{i k}^{ \pm}(u) .
$$

\subsection{Bethe vectors for $U(R)$}

The construction below was borrowed from [TV1]. We will need only one $L$-operator, say $L^{+}(z)$, which we denote simply as $L(z)$. Let $M$ be a nonnegative integer. Let $L^{(k)}(z) \in\left(\mathbb{C}^{N}\right)^{\otimes M}$ be $L$-operator acting as $L(z)$ in $k$-th tensor factor of the product $\left(\mathbb{C}^{N}\right)^{\otimes M}$ and as identity operator in all other factors. Consider a series in $M$ variables

$$
\mathbb{T}_{[M]}\left(u_{1}, \ldots, u_{M}\right)=L^{(1)}\left(u_{1}\right) \cdots L^{(M)}\left(u_{M}\right) \cdot \mathbb{R}^{(M, \ldots, 1)}\left(u_{M}, \ldots, u_{1}\right)
$$


with coefficients in $\left(\operatorname{End}\left(\mathbb{C}^{N}\right)\right)^{\otimes M} \otimes U_{q}\left(\mathfrak{b}_{+}\right)$, where

$$
\mathbb{R}^{(M, \ldots, 1)}\left(u_{M}, \ldots, u_{1}\right)=\prod_{1 \leq i<j \leq M} R^{(j i)}\left(u_{j}, u_{i}\right)
$$

The factor $R^{(j i)}$ is to the left of the factor $R^{(m l)}$ if $j>m$, or $j=m$ and $i>l$ in the ordered product of $R$-matrices (2.7). Note, that due to the Yang-Baxter equation for $L$-operators, the element (2.6) can be rewritten in the inverse order

$$
\mathbb{T}_{[M]}\left(u_{1}, \ldots, u_{M}\right)=\mathbb{R}^{(M, \ldots, 1)}\left(u_{M}, \ldots, u_{1}\right) \cdot L^{(M)}\left(u_{M}\right) \cdots L^{(1)}\left(u_{1}\right) .
$$

Let us consider a set of colored variables

$$
\bar{t}_{\bar{n}}=t_{1}^{1}, \ldots, t_{n_{1}}^{1}, \quad t_{1}^{2}, \ldots, t_{n_{2}}^{2}, \quad \ldots, t_{1}^{N-1}, \ldots, t_{n_{N-1}}^{N-1}
$$

labeled by a sequence of the non-negative integers $\bar{n}=\left\{n_{1}, n_{2}, \ldots, n_{N-1}\right\}, \bar{n} \in$ $\mathbb{Z}_{\geq 0}^{N-1},|\bar{n}|=n_{1}+\cdots+n_{N-1}$. The upper index $i$ in $t_{j}^{i}$ corresponds to the color of this variable. Following [TV1] set

$$
\begin{gathered}
\mathbb{B}_{\bar{n}}\left(\bar{t}_{\bar{n}}\right)=\prod_{a=1}^{N-1} \prod_{1 \leq i<j \leq n_{a}} \frac{t_{i}^{a}-t_{j}^{a}}{q^{-1} t_{i}^{a}-q t_{j}^{a}} \\
\times\left((\operatorname{tr})^{\otimes|\bar{n}|} \otimes \mathrm{id}\right)\left(\mathbb{T}_{[|\bar{n}|]}\left(t_{1}^{1}, \ldots, t_{n_{1}}^{1} ; \ldots ; t_{1}^{N-1}, \ldots, t_{n_{N-1}}^{N-1}\right)\right. \\
\left.\times E_{21}^{\otimes n_{1}} \otimes \cdots \otimes E_{N, N-1}^{\otimes n_{N-1}} \otimes 1\right) .
\end{gathered}
$$

Here tr: $\operatorname{End}\left(\mathbb{C}^{N}\right) \rightarrow \mathbb{C}$ is the standard trace map.

Let $S_{\bar{n}}=S_{n_{1}} \times \cdots \times S_{n_{N-1}}$ be the direct product of the symmetric groups. The group $S_{\bar{n}}$ acts naturally on the functions of $|\bar{n}|$ colored variables by permutations of the variables of the same colour. If $\sigma=\sigma^{1} \times \cdots \times \sigma^{N-1} \in S_{\bar{n}}$, then we have

Proposition 2.1.

$$
\mathbb{B}_{\bar{n}}\left(\bar{t}_{\bar{n}}\right)=\mathbb{B}_{\bar{n}}\left(\sigma_{\bar{t}_{\bar{n}}}\right)
$$

where

$$
\sigma_{\bar{t}_{\bar{n}}}=\left(t_{\sigma^{1}(1)}^{1}, \ldots, t_{\sigma^{1}\left(n_{1}\right)}^{1} ; \ldots ; t_{\sigma^{N-1}(1)}^{N-1}, \ldots, t_{\sigma^{N-1}\left(n_{N-1}\right)}^{N-1}\right) .
$$

Proof is based on the relation

$$
\begin{aligned}
& P^{(i, i+1)} R^{(i, i+1)}\left(u_{i}, u_{i+1}\right) \mathbb{T}_{[M]}\left(u_{1}, \ldots, u_{i}, u_{i+1}, \ldots, u_{M}\right) \\
& \quad=\mathbb{T}_{[M]}\left(u_{1}, \ldots, u_{i+1}, u_{i}, \ldots, u_{M}\right) P^{(i+1, i)} R^{(i+1, i)}\left(u_{i+1}, u_{i}\right)
\end{aligned}
$$

where permutation operator $P^{(i, i+1)}$ is defined as usual by $P^{(12)}=\sum E_{a b} \otimes E_{b a}$.

We call a vector $v$ a weight singular vector if it is annihilated by any mode of the matrix elements $L_{i j}[n], i>j$, and is an eigenvector for the diagonal elements $L_{i i}(z)$ :

$$
L_{i j}(z) \cdot v=0, \quad i>j, \quad L_{i i}(z) \cdot v=\Lambda_{i}(z) v
$$

where $\Lambda_{i}(z)$ is a meromorphic function, decomposed into a series over $z^{-1}$.

Let $V$ be $U_{q}\left(\mathfrak{b}_{+}\right)$-module generated by the singular vector $v(2.13)$. 
Definition 2.2. The $V$-valued function

$$
\mathbb{B}_{\bar{n}}\left(\bar{t}_{\bar{n}}\right) v
$$

is a weight function or off-shell Bethe vectors parametrized by the set of parameters $\bar{t}_{\bar{n}}(2.9)$.

In case of the simplest algebra $U_{q}\left(\widehat{\mathfrak{g l}}_{2}\right)$ the element $(2.10)$ is equal to the product $L_{12}\left(t_{1}\right) \cdots L_{12}\left(t_{n}\right)$ and the weight function (2.14) is defined by the vector, which is annihilated by the element $L_{21}\left(t_{1}\right) v=0$. A $V$-valued function $L_{12}\left(t_{1}\right) \cdots L_{12}\left(t_{n}\right) v$ is a Bethe vector or eigen-vector of the transfer operator $L_{11}(u)+L_{22}(u)$, if the parameters $t_{i}$ satisfy Bethe equations. In this paper parameters $t_{i}$ are free and this is a reason to call $V$-valued function (2.14) as off-shell Bethe vectors.

In case of the higher rank algebras a weight function defined by the relation (2.14) satisfies hierarchical relations appeared in the hierarchical or nested Bethe ansatz. This method was proposed in $[\mathrm{KR}]$ and was developed for the diagonalization of the $\mathfrak{g l}(N)$ invariant transfer matrices. The cornerstone of this method was hierarchical relations between Bethe vectors for the algebras $\mathfrak{g l}(N)$ and $\mathfrak{g l}(N-1)$. The weight function (2.14) is an effective solution to these hierarchical relations. The goal of this note is to reformulate this formula in a different language, using the current realization of the algebra $U_{q}\left(\widehat{\mathfrak{g l}}_{N}\right)$.

To show that the $V$-valued function (2.14) satisfies the hierarchical relations, one has to use evaluation homomorphism of the algebra $U_{q}\left(\widehat{\mathfrak{g l}}_{N}\right)$ onto $U_{q}\left(\mathfrak{g l}_{N}\right)$. There exist a class of the weight functions such that the hierarchical relations can be written without knowledge of this homomorphism. At the end of the paper we will prove that the identical hierarchical relations can be obtained using certain projections in the current realization of $U_{q}\left(\widehat{\mathfrak{g l}}_{N}\right)[\mathrm{D}]$.

\subsection{Recursion relation for the weight function}

Let us consider the element (2.10) depending on the specific set of colored variables

$$
\bar{t}=\left\{t^{1}, t^{2}, \ldots, t^{N-2}, t^{N-1}\right\}
$$

when $n_{1}=n_{2}=\cdots=n_{N-1}=1$. Denote the corresponding weight function as $\mathbb{B}_{[N-1]}(t) v$. It is nontrivial for the symmetric tensor products of the $U_{q}\left(\widehat{\mathfrak{g l}}_{N}\right)$ vector representations only. Here we will find a recursion relation for the weight function $\mathbb{B}_{[N-1]}(t) v$.

For $m=1, \ldots, N-1$ we introduce a set of embedding of the algebras $U_{q}\left(\widehat{\mathfrak{g l}}_{N-m}\right)$ into $U_{q}\left(\widehat{\mathfrak{g l}}_{N}\right)$

$$
\psi^{(m)}\left(L_{i j}^{(N-m)}(t)\right)=L_{i+m, j+m}^{(N)}, \quad i, j=1, \ldots, N-m .
$$

Let

$$
\begin{aligned}
& \mathbb{B}_{[N-m]}\left(t^{m}, t^{m+1}, \ldots, t^{N-1}\right)=\operatorname{tr}_{m, m+1, \ldots, N-1}\left(\mathbb{R}^{(N-1, \ldots, m)}\left(t^{N-1}, \ldots, t^{m}\right)\right. \\
& \left.\quad \times L^{(N-1)}\left(t^{N-1}\right) \cdots L^{(m+1)}\left(t^{m+1}\right) L^{(m)}\left(t^{m}\right) E_{N, N-1}^{(N-1)} \cdots E_{m+2, m+1}^{(m+1)} E_{m+1, m}^{(m)}\right)
\end{aligned}
$$


be the elements of $U_{q}\left(\mathfrak{b}_{+}^{(N-m)}\right) \in U_{q}\left(\widehat{\mathfrak{g l}}_{N-m}\right)$. We set also

$$
\mathbb{B}_{[0]}(\cdot) \equiv 1 \quad \text { and } \quad \mathbb{B}_{[1]}\left(t^{N-1}\right)=L_{N-1, N}\left(t^{N-1}\right) .
$$

Using definition (2.10) one may calculate an element

$$
\begin{aligned}
\mathbb{B}_{[2]}\left(t^{N-2}, t^{N-1}\right) & =L_{N-1, N}\left(t^{N-1}\right) L_{N-2, N-1}\left(t^{N-2}\right) \\
& +\frac{\left(q-q^{-1}\right) t^{N-1}}{t^{N-1}-t^{N-2}} L_{N-2, N}\left(t^{N-1}\right) L_{N-1, N-1}\left(t^{N-2}\right) .
\end{aligned}
$$

We claim that the following recursion relation is valid

\section{Proposition 2.3.}

$$
\begin{aligned}
\mathbb{B}_{[N-1]}\left(t^{1}, \ldots, t^{N-1}\right) v & =\sum_{m=2}^{N} \mathbb{B}_{[N-m]}\left(t^{m}, \ldots, t^{N-1}\right) L_{1, m}\left(t^{m-1}\right) v \\
& \times \prod_{j=2}^{m-1} \Lambda_{j}\left(t^{j-1}\right) \frac{\left(q-q^{-1}\right) t^{j}}{t^{j}-t^{j-1}},
\end{aligned}
$$

where the vectors $L_{1, m}\left(t^{m-1}\right) v$ are singular for the subalgebras $U_{q}\left(\widehat{\mathfrak{g l}}_{N-m}\right)$, so the recursion relation (2.18) can be considered as the relation between the corresponding $U_{q}\left(\widehat{\mathfrak{g l}}_{N-m}\right)$ weight functions.

Proof can be done by the recurrent application of the following

\section{Lemma 2.4.}

$$
\begin{aligned}
& \mathbb{B}_{[N-1]}\left(t^{1}, t^{2}, \ldots, t^{N-1}\right) v=\left[\mathbb{B}_{[N-2]}\left(t^{2}, \ldots, t^{N-1}\right) \cdot L_{12}\left(t^{1}\right)\right. \\
& \quad+\operatorname{tr}_{2, \ldots, N-1}\left(\mathbb{R}^{(N-1, \ldots, 2)}\left(t^{N-1}, \ldots, t^{2}\right) L^{(N-1)}\left(t^{N-1}\right) \cdots L^{(2)}\left(t^{2}\right)\right. \\
& \left.\left.\quad \times E_{N, N-1}^{(N-1)} \cdots E_{4,3}^{(3)} E_{3,1}^{(2)}\right) L_{22}\left(t^{1}\right) \frac{\left(q-q^{-1}\right) t^{2}}{t^{2}-t^{1}}\right] v .
\end{aligned}
$$

Proof of this lemma is based on the calculation of the trace over first $N$-dimensional space $\mathbb{C}^{N}$ in the element $\mathbb{B}_{[N-1]}\left(t^{1}, t^{2}, \ldots, t^{N-1}\right)$. Due to the upper triangular form of the $L$-operator (2.13) acting on the singular weight vector the element $\mathbb{B}_{[N-1]}(t)$ can be written as the sum of two terms

$$
\begin{aligned}
& \operatorname{tr}_{2, \ldots, N-1}\left(\operatorname{tr}_{1}\left(R^{(21)}\left(t^{2}, t^{1}\right) \cdots R^{(N-1,1)}\left(t^{N-1}, t^{1}\right) E_{11}^{(1)}\right) \cdot X \cdot L_{12}\left(t^{1}\right)\right. \\
& \left.+\operatorname{tr}_{1}\left(R^{(21)}\left(t^{2}, t^{1}\right) \cdots R^{(N-1,1)}\left(t^{N-1}, t^{1}\right) E_{21}^{(1)}\right) \cdot X \cdot L_{22}\left(t^{1}\right)\right)
\end{aligned}
$$

where

$$
X=\mathbb{B}_{[N-2]}\left(t^{2}, \ldots, t^{N-1}\right) .
$$

The Yang-Baxter equation (2.2) for $R$-matrices was used in order to rearrange them in the product $(2.7)$ :

$$
\begin{aligned}
& \mathbb{R}^{(N-1, \ldots, 2,1)}\left(t^{N-1}, \ldots, t^{2}, t^{1}\right) \\
& \quad=R^{2,1}\left(t^{2}, t^{1}\right) \cdots R^{N-1,1}\left(t^{N-1}, t^{1}\right) \mathbb{R}^{(N-1, \ldots, 2)}\left(t^{N-1}, \ldots, t^{2}\right) .
\end{aligned}
$$


We may calculate the traces over first space $\mathbb{C}^{N}$ in (2.19) using

$$
\operatorname{tr}_{1}\left(R^{(21)}\left(t^{2}, t^{1}\right) \cdots R^{(N-1,1)}\left(t^{N-1}, t^{1}\right) E_{11}^{(1)}\right)=\mathbf{1}^{(2)} \mathbf{1}^{(3)} \cdots \mathbf{1}^{(N-1)}+\cdots
$$

and

$$
\begin{aligned}
\operatorname{tr}_{1}\left(R^{(21)}\left(t^{2}, t^{1}\right) \cdots R^{(N-1,1)}\left(t^{N-1}, t^{1}\right) E_{21}^{(1)}\right) & \\
= & \frac{\left(q-q^{-1}\right) t^{2}}{t^{2}-t^{1}} E_{21}^{(2)} \mathbf{1}^{(3)} \cdots \mathbf{1}^{(N-1)}+\cdots,
\end{aligned}
$$

where by dots we denoted the terms which are annihilated by the trace over the rest $N$-dimensional spaces. Note that we may move matrix $E_{21}^{(2)}$ to the right under the trace over the second space $\mathbb{C}^{N}$ in the second term of (2.19) and use the product property $E_{32}^{(2)} \cdot E_{21}^{(2)}=E_{31}^{(2)}$. Lemma is proved.

Return to the proof of the Proposition 2.3. The vector $L_{12}\left(t^{1}\right) v$ is a $U_{q}\left(\widehat{\mathfrak{g l}}_{N-1}\right)$ singular vector if $v$ is a $U_{q}\left(\widehat{\mathfrak{g l}}_{N}\right)$ singular vector and the subalgebra $U_{q}\left(\widehat{\mathfrak{g l}}_{N-1}\right)$ is embedded into $U_{q}\left(\widehat{\mathfrak{g l}}_{N}\right)$ by the map $\psi^{(1)}(2.16)$. The action of the element $L_{22}\left(t^{2}\right)$ onto weight singular vector $v$ is diagonal and it remains a singular $U_{q}\left(\widehat{\mathfrak{g l}}_{N}\right)$ vector. Taking this into account we may proceed further calculating the vector

$$
\operatorname{tr}_{2, \ldots, N-1}\left(\mathbb{R}^{(N-1, \ldots, 2)}\left(t^{N-1}, \ldots, t^{2}\right) L^{(N-1)}\left(t^{N-1}\right) \cdots L^{(2)}\left(t^{2}\right) E_{N, N-1}^{(N-1)} \cdots E_{3,1}^{(2)}\right) v
$$

in the same way as we did it proving Lemma 2.4. Applying the same procedure several times we obtain the recursion relation formulated in the Proposition 2.3.

\section{The current realization of $U_{q}\left(\widehat{\mathfrak{g l}}_{N}\right)$}

It was shown in $[\mathrm{DF}]$ that the algebra $U(R)[\mathrm{RS}]$ is isomorphic to the algebra $U_{q}\left(\widehat{\mathfrak{g l}}_{N}\right)$ in the current realization $[\mathrm{D}]^{1}$. The algebra $U_{q}\left(\widehat{\mathfrak{g l}}_{N}\right)$ in the current realization is generated by the modes of the Cartan currents

$$
k_{i}^{ \pm}(z)=\sum_{m \geq 0} k_{i}^{ \pm}[ \pm m] z^{\mp m}, \quad k_{i}^{+}[0] k_{i}^{-}[0]=1,
$$

$i=1, \ldots, N$ and by the modes of the currents

$$
E_{i}(z)=\sum_{n \in \mathbb{Z}} E_{i}[n] z^{-n}, \quad F_{i}(z)=\sum_{n \in \mathbb{Z}} F_{i}[n] z^{-n},
$$

$i=1, \ldots, N-1$. These currents satisfy the commutation relations

$$
\begin{aligned}
& \left(q^{-1} z-q w\right) E_{i}(z) E_{i}(w)=E_{i}(w) E_{i}(z)\left(q z-q^{-1} w\right), \\
& (z-w) E_{i}(z) E_{i+1}(w)=E_{i+1}(w) E_{i}(z)\left(q^{-1} z-q w\right), \\
& \left(q z-q^{-1} w\right) F_{i}(z) F_{i}(w)=F_{i}(w) F_{i}(z)\left(q^{-1} z-q w\right), \\
& \left(q^{-1} z-q w\right) F_{i}(z) F_{i+1}(w)=F_{i+1}(w) F_{i}(z)(z-w),
\end{aligned}
$$

${ }^{1}$ We will always understand the algebra $U_{q}\left(\widehat{\mathfrak{g l}}_{N}\right)$ as the algebra with zero central charge and dropped gradation operator. 


$$
\begin{gathered}
k_{i}^{ \pm}(z) F_{i}(w)\left(k_{i}^{ \pm}(z)\right)^{-1}=\frac{q^{-1} z-q w}{z-w} F_{i}(w), \\
k_{i+1}^{ \pm}(z) F_{i}(w)\left(k_{i+1}^{ \pm}(z)\right)^{-1}=\frac{q z-q^{-1} w}{z-w} F_{i}(w), \\
k_{i}^{ \pm}(z) F_{j}(w)\left(k_{i}^{ \pm}(z)\right)^{-1}=F_{j}(w) \quad \text { if } i \neq j, j+1, \\
k_{i}^{ \pm}(z) E_{i}(w)\left(k_{i}^{ \pm}(z)\right)^{-1}=\frac{z-w}{q^{-1} z-q w} E_{i}(w), \\
k_{i+1}^{ \pm}(z) E_{i}(w)\left(k_{i+1}^{ \pm}(z)\right)^{-1}=\frac{z-w}{q z-q^{-1} w} E_{i}(w), \\
k_{i}^{ \pm}(z) E_{j}(w)\left(k_{i}^{ \pm}(z)\right)^{-1}=E_{j}(w) \text { if } i \neq j, j+1, \\
{\left[E_{i}(z), F_{j}(w)\right]=\delta_{i, j} \delta(z / w)\left(q-q^{-1}\right)\left(k_{i}^{+}(z) / k_{i+1}^{+}(z)-k_{i}^{-}(w) / k_{i+1}^{-}(w)\right)}
\end{gathered}
$$

and the Serre relations

$$
\begin{aligned}
\operatorname{Sym}_{z_{1}, z_{2}}\left(E_{i}\left(z_{1}\right) E_{i}\left(z_{2}\right) E_{i \pm 1}(w)\right. & -\left(q+q^{-1}\right) E_{i}\left(z_{1}\right) E_{i \pm 1}(w) E_{i}\left(z_{2}\right) \\
& \left.+E_{i \pm 1}(w) E_{i}\left(z_{1}\right) E_{i}\left(z_{2}\right)\right)=0 \\
\operatorname{Sym}_{z_{1}, z_{2}}\left(F_{i}\left(z_{1}\right) F_{i}\left(z_{2}\right) F_{i \pm 1}(w)\right. & -\left(q+q^{-1}\right) F_{i}\left(z_{1}\right) F_{i \pm 1}(w) F_{i}\left(z_{2}\right) \\
& \left.+F_{i \pm 1}(w) F_{i}\left(z_{1}\right) F_{i}\left(z_{2}\right)\right)=0 .
\end{aligned}
$$

All these relations between currents should be understood as the relations between modes of the currents and the latter should be treated as formal series.

To construct an isomorphism between the algebra $U(R)$ and current realization of $U_{q}\left(\widehat{\mathfrak{g l}}_{N}\right)$, one has to decompose $L$-operators into Gauss coordinates

$$
\begin{aligned}
L^{ \pm}(z)=\left(\begin{array}{cccc}
1 & F_{1,2}^{ \pm}(z) & \cdots & F_{1, N}^{ \pm}(z) \\
0 & 1 & \ddots & \vdots \\
& 0 & \ddots & F_{N-1, N}^{ \pm}(z) \\
0 & 1 &
\end{array}\right)\left(\begin{array}{ccccc}
k_{1}^{ \pm}(z) & & & \\
& \ddots & 0 & \\
& & 0 & \ddots & \\
& & & & \\
& & & & \\
& & & \\
E_{2,1}^{ \pm}(z) & \ddots & 0 & \\
\vdots & \ddots & 1 & 0 \\
E_{N, 1}^{ \pm}(z) & \cdots & E_{N, N-1}^{ \pm}(z) & 1
\end{array}\right)
\end{aligned}
$$

and identify

$$
E_{i}(z)=E_{i+1, i}^{+}(z)-E_{i+1, i}^{-}(z), \quad F_{i}(z)=F_{i, i+1}^{+}(z)-F_{i, i+1}^{-}(z) .
$$

Diagonal Gauss coordinates of $L$-operators are the same as Cartan currents $k_{i}^{ \pm}(z)$ and denoted by the same letter. The relations between Gauss coordinates $F_{i, j}^{ \pm}(z)$, $E_{j, i}^{ \pm}(z)$ of $L$-operators for $j>i+1$ and currents $F_{i}(z), E_{i}(z)$ were not discussed in the paper $[\mathrm{DF}]$. These relations will be considered below (see Subsection 4.1). 
In [D] another current Hopf structure was defined for the algebra $U_{q}\left(\widehat{\mathfrak{g l}}_{N}\right)$

$$
\begin{aligned}
\Delta^{(D)}\left(E_{i}(z)\right) & =E_{i}(z) \otimes 1+k_{i}^{-}(z)\left(k_{i+1}^{-}(z)\right)^{-1} \otimes E_{i}(z), \\
\Delta^{(D)}\left(F_{i}(z)\right) & =1 \otimes F_{i}(z)+F_{i}(z) \otimes k_{i}^{+}(z)\left(k_{i+1}^{+}(z)\right)^{-1}, \\
\Delta^{(D)}\left(k_{i}^{ \pm}(z)\right) & =k_{i}^{ \pm}(z) \otimes k_{i}^{ \pm}(z) .
\end{aligned}
$$

The relation between the standard (2.5) and current (3.27) coproducts for the quantum affine algebras was explained in [KhT].

\subsection{Borel subalgebras in $U_{q}\left(\widehat{\mathfrak{g l}}_{N}\right)$ and projections}

Standard Borel subalgebras $U_{q}\left(\mathfrak{b}_{ \pm}\right)$of the algebra $U_{q}\left(\widehat{\mathfrak{g l}}_{N}\right)$ are generated by the modes of the $L$-operators $L^{ \pm}(z)$ respectively.

Another type of Borel subalgebras are naturally associated with the current realization of $U_{q}\left(\widehat{\mathfrak{g l}}_{N}\right)$. Denote by $U_{F}$ subalgebra of $U_{q}\left(\widehat{\mathfrak{g l}}_{N}\right)$ generated by the modes of the currents $F_{i}[n], k_{j}^{+}[m], i=1, \ldots, N-1, j=1, \ldots, N, n \in \mathbb{Z}, m \geq 0$. Denote by $U_{E}$ subalgebra of $U_{q}\left(\widehat{\mathfrak{g l}}_{N}\right)$ generated by the modes of the currents $E_{i}[n]$, $k_{j}^{-}[-m], i=1, \ldots, N-1, j=1, \ldots, N, n \in \mathbb{Z}, m \geq 0$. Introduce also subalgebra $U_{F}^{\prime}=U_{F} \backslash\left\{k_{j}\right\}$ and $U_{E}^{\prime}=U_{E} \backslash\left\{k_{j}\right\}$, where $k_{j}=k_{j}^{+}[0]=k_{j}^{-}[0]^{-1}$ are zero modes of the Cartan currents $k_{j}^{ \pm}(z)$.

Define intersections of the different type of Borel subalgebras by the following formulas

$$
\begin{array}{rlrl}
U_{f}^{-} & =U_{F}^{\prime} \cap U_{q}\left(\mathfrak{b}_{-}\right), & & U_{F}^{+}=U_{F} \cap U_{q}\left(\mathfrak{b}_{+}\right), \\
U_{e}^{+}=U_{E}^{\prime} \cap U_{q}\left(\mathfrak{b}_{+}\right), & U_{E}^{-}=U_{E} \cap U_{q}\left(\mathfrak{b}_{-}\right) .
\end{array}
$$

It follows from the definition of these intersections that subalgebras $U_{f}^{-}$and $U_{e}^{+}$ are generated by the non-positive $F_{i}[n \leq 0]$ and non-negative $E_{i}[n \geq 0]$ modes of the currents $F_{i}(z)$ and $E_{i}(z)$ respectively. On the contrary, description of the subalgebras $U_{F}^{+}$and $U_{E}^{-}$is more complicated. Their structure are given by the following

Proposition 3.1. Subalgebras $U_{F}^{+}$and $U_{E}^{-}$are generated by the modes of the currents $k_{i}^{+}(z)$ and $k_{i}^{-}(z), i=1, \ldots, N$ and the sets of elements

$$
F_{i, j}[1]=S_{F_{i}[0]} \cdot S_{F_{i+1}[0]} \cdots S_{F_{j-2}[0]} \cdot F_{j-1}[1]
$$

for $U_{F}^{+}$and

$$
E_{j, i}[-1]=S_{E_{j-1}[0]} \cdot S_{E_{j-2}[0]} \cdots S_{E_{i+1}[0]} \cdot E_{i}[-1]
$$

for $U_{E}^{-}$, where $1 \leq i<j \leq N$ and $S_{A} \cdot B=B A-q A B$.

For the proof of this Proposition see $[\mathrm{KhP}]$. Note that when $j=i+1$ in the formulas (3.30) and (3.31) the elements $F_{i, i+1}[1]$ and $E_{i+1, i}[-1]$ coincide with the modes of the currents $F_{i}[1]$ and $E_{i}[-1]$ respectively. We shall call $S_{F_{i}[0]}$ the screening operators.

A theory of bi-orthogonal decompositions of the Hopf algebras was developed in the papers $[\mathrm{ER}, \mathrm{DKhP}, \mathrm{EKhP}]$. In application to the current Borel subalgebras 
$U_{F}$ and $U_{E}$ equipped with coproduct $\Delta^{(D)}$ this theory reads as follows. There are two pairs of decompositions: the first one is $U_{F}=U_{F}^{+} U_{f}^{-}$and $U_{E}=U_{E}^{-} U_{e}^{+}$, and the second $-U_{E}=U_{e}^{+} U_{E}^{-}$and $U_{F}=U_{f}^{-} U_{F}^{+}$.

Together with these decompositions one can introduce projections onto intersections (3.28) and (3.29) [ER]. In what follows we will consider only the current Borel subalgebra $U_{F}$ and its decomposition $U_{F}=U_{f}^{-} \cdot U_{F}^{+}$. According to the general theory, we introduce the projection operators $P^{ \pm}$acting onto $U_{F}$ as

$$
P^{+}\left(f_{-} f_{+}\right)=\varepsilon\left(f_{-}\right) f_{+}, \quad P^{-}\left(f_{-} f_{+}\right)=f_{-} \varepsilon\left(f_{+}\right),
$$

where $\varepsilon$ is a co-unit map associated with the coproduct $(3.27) \varepsilon\left(F_{i}(z)\right)=0$, $\varepsilon\left(k_{i}(z)\right)=1$. We will use below only the operator $P^{+}$which we denote as $P$.

An important property of the projection was proved in $[\mathrm{EKhP}]$. For any $f \in U_{f}$

$$
\Delta(P(f)) v_{1} \otimes v_{2}=(P \otimes P)\left(\Delta^{(D)}(f)\right) v_{1} \otimes v_{2},
$$

where $\Delta$ and $\Delta^{(D)}$ are standard (2.5) and current (3.27) coproducts respectively and $v_{1,2}$ are two weight singular vectors. This property of the projection trivialize the verification of the coproduct properties of the weight functions defined via projections (see details in $[\mathrm{EKhP}]$ ).

\section{Weight function as the projection}

The main result of this paper is the proof of coincidence of the weight function $\mathbb{B}_{[N-1]}\left(\bar{t}_{\bar{n}}\right) v$ given in terms of $L$-operators and as the projection of the certain product of $U_{q}\left(\widehat{\mathfrak{g l}}_{N}\right)$ currents. We formulate this result as the following

\section{Theorem 1.}

$$
\mathbb{B}_{[N-1]}\left(t^{1}, \ldots, t^{N-1}\right) v=P\left(F_{N-1}\left(t^{N-1}\right) \cdots F_{1}\left(t^{1}\right)\right) \prod_{j=1}^{N-1} k_{j+1}\left(t^{j}\right) v .
$$

Proof will follow from observation that the projection $P\left(F_{N-1}\left(t^{N-1}\right) \cdots F_{1}\left(t^{1}\right)\right)$ and the action of the element $\mathbb{B}_{[N-1]}(t)$ onto singular vector satisfy identical recursion relation on the rank $N$. Before starting this proof we need to establish the relation between Gauss coordinates $L_{i j}(t), j>i+1$ and projections of the currents.

\subsection{More on Ding-Frenkel isomorphism}

Gauss coordinates $F_{i, j}^{+}(z)$ for $j>i+1$ will be identified in this subsection with projections of composed currents. For a convenience we will sometimes denote currents $F_{i}(z)$ as $F_{i, i+1}(z)$. Gauss coordinates will be denoted by the same letter $F_{i, j}^{+}, j>i$, but always will have upper superscript "+". Notation $F_{i, j}$ will always denote the composed current defined below for $j>i+1$.

A notion of the composed currents for the quantum affine algebras was introduced in [DKh]. These currents belong to certain completion $\bar{U}_{f}$ of the subalgebra 
of modes $F_{i}[n], n \in \mathbb{Z}$. This completion is defined by the extension of this subalgebra by the elements which are infinite sums of monomials composed from the ordered products of modes. It was shown in [DKh] that such elements are correctly defined in the category of the highest degree representations of $U_{q}\left(\widehat{\mathfrak{g l}}_{N}\right)$. In this category, the products of currents have simple poles at the points prescribed by the commutation relations between them. The composed currents are defined inductively as residues at these poles (see details in [DKh, DKhP]). Using results of these papers we introduce $U_{q}\left(\widehat{\mathfrak{g l}}_{N}\right)$ composed currents by the following

\section{Proposition 4.1.}

(i) The composed currents $F_{i, j}(t), 2<i+1<j \leq N$ can be defined inductively as residues of the products of the preceding currents and equal to

$$
F_{i, j}(t)=\left(q-q^{-1}\right)^{j-i-1} F_{i}(t) F_{i+1}(t) \cdots F_{j-2}(t) F_{j-1}(t) .
$$

(ii) Projections of these composed currents can also be defined inductively by $q$ commutators with zero-modes

$$
P\left(F_{i, j}(t)\right)=S_{F_{i}[0]} \cdot S_{F_{i+1}[0]} \cdots S_{F_{j-2}[0]}\left(F_{j-1, j}^{+}(t)\right) .
$$

Proof. Here we only sketch the proof. The composed currents are defined inductively from the basic currents $F_{i, i+1}(z)$ using the relations

$$
\begin{aligned}
F_{i, j}(z) & =-\underset{w=z}{\operatorname{res}} F_{s+1, j}(z) F_{i, s+1}(w) \frac{d w}{w} \\
& =\oint_{C_{0}} F_{s+1, j}(z) F_{i, s+1}(w) \frac{d w}{w}-\oint_{C_{\infty}} \frac{q^{-1}-q z / w}{1-z / w} F_{i, s+1}(w) F_{s+1, j}(z) \frac{d w}{w}
\end{aligned}
$$

for any $s$ such that $i \leq s<j-1$ and $C_{0}$ and $C_{\infty}$ are small contours around 0 and $\infty$ respectively. Positions of the poles in the product $F_{s+1, j}(z) F_{i, s+1}(w)$ are defined by the commutation relations

$$
\left(q^{-1} t-q s\right) F_{i, s+1}(t) F_{s+1, j}(s)=(t-s) F_{s+1, j}(s) F_{i, s+1}(t) .
$$

Calculating residues by means of these commutation relations we prove the statement (i) of the Proposition 4.1.

On the other hand we may calculate formal integrals in the second line of (4.37) to obtain

$$
\begin{aligned}
F_{i, j}(z)=F_{s+1, j}(z) F_{i, s+1}[0]-q F_{i, s+1}[0] F_{s+1, j}(z) & \\
& -\left(q^{-1}-q\right) \sum_{k \geq 0} F_{i, s+1}[-k] F_{s+1, j}(z) z^{k}
\end{aligned}
$$

where $F_{i, s+1}[-k]$ means non-positive modes of the current $F_{i, s+1}(z)$.

Let us consider equality (4.39) for $s=i$ and apply projection $P^{+}$to both sides of this relation. According to our notations, currents $F_{i, i+1}(z) \equiv F_{i}(z)$ and 
modes $F_{i, i+1}[k \leq 0] \in U_{f}^{-}$. Due to the formulas (3.32) the projection $P$ kills the semi-infinite sum in the right-hand side of (4.39) and we get the equality:

$$
\begin{aligned}
P\left(F_{i, j}(z)\right) & =P\left(F_{i+1, j}(z)\right) F_{i, i+1}[0]-q F_{i, i+1}[0] P\left(F_{i+1, j}(z)\right) \\
& =S_{F_{i, i+1}[0]}\left(P\left(F_{i+1, j}(z)\right)\right) .
\end{aligned}
$$

In order to obtain this relation we used the fact of commutativity of the projections and the screening operators $S_{F_{i}[0]}(F), \forall F \in U_{F}^{\prime}$. Repeating assertion (4.40) several times we prove the statement (ii) of the Proposition 4.1.

We are ready now to prove the identification formulas for the projection of the composed currents and the Gauss coordinates $F_{i, j}^{+}(z), i+1<j$. Actually, it is sufficient to verify this identification only for the action onto weight singular vectors.

Proposition 4.2. The actions of projections of the composed currents and higher Gauss coordinates onto a weight singular vector coincide

$$
P\left(F_{i, j}(t)\right) v=\left(q-q^{-1}\right)^{j-i-1} F_{i, j}^{+}(t) v, \quad i+1<j .
$$

Proof of this proposition is based on comparing the commutation relations between Gauss coordinates which follow from the $R L L$-relations and commutation relations between projections of composed currents. We start by considering the commutation relations for the following elements of $L$-operators:

$$
\begin{aligned}
& (s-t) L_{j-1, j}(s) L_{i, j-1}(t)+s\left(q-q^{-1}\right) L_{i, j}(s) L_{j-1, j-1}(t) \\
& \quad=(s-t) L_{i, j-1}(t) L_{j-1, j}(s)+t\left(q-q^{-1}\right) L_{i, j}(t) L_{j-1, j-1}(s) .
\end{aligned}
$$

Each matrix element of $L$-operators which enters to the commutation relation (4.42) is a combination of Gauss coordinates, for example,

$$
L_{j, j}(t)=k_{j}(t)+\sum_{n=j+1}^{N} F_{j, n}^{+}(t) k_{n}(t) E_{n, j}^{+}(t) .
$$

Note, that $L_{j, j}(t) v=k_{j}(t) v$, where $v$ is a weight singular vector (2.13).

Let us apply both sides of the equality (4.42) to the vector $v$. As result we obtain the commutation relations

$$
\begin{aligned}
& \left((s-t) F_{j-1, j}^{+}(s) F_{i, j-1}^{+}(t)+s\left(q-q^{-1}\right) F_{i, j}^{+}(s)\right) v \\
& =\left(\left(q s-q^{-1} t\right) F_{i, j-1}^{+}(t) F_{j-1, j}^{+}(s)+t\left(q^{-1}-q\right) F_{i, j-1}^{+}(t) F_{j-1, j}^{+}(t)\right. \\
& \left.\quad+t\left(q-q^{-1}\right) F_{i, j}^{+}(t)\right) v .
\end{aligned}
$$

On the other hand let us write the commutation relations between currents (4.38) in terms of modes

$$
\begin{aligned}
F_{s+1, j}[n+1] F_{i, s+1}[p]-F_{s+1, j}[n] F_{i, s+1}[p+1] \\
=q F_{i, s+1}[p] F_{s+1, j}[n+1]-q^{-1} F_{i, s+1}[p+1] F_{s+1, j}[n]
\end{aligned}
$$

and consider this commutation relations at the values $i+1=s=j-1=m$. 
From the commutation relations (4.44) we may obtain the commutation relations for the projections $P\left(F_{m-1, m}(t)\right)=F_{m-1, m}^{+}(t)$ and $P\left(F_{m, m+1}(s)\right)=$ $F_{m, m+1}^{+}(s)$ in the form

$$
\begin{aligned}
&(s-t) F_{m, m+1}^{+}(s) F_{m-1, m}^{+}(t)+s P\left(F_{m-1, m+1}(s)\right) \\
&=\left(q s-q^{-1} t\right) F_{m-1, m}^{+}(t) F_{m, m+1}^{+}(s)+t\left(q^{-1}-q\right) F_{m-1, m}^{+}(t) F_{m, m+1}^{+}(t) \\
&+t P\left(F_{m-1, m+1}(t)\right) .
\end{aligned}
$$

Applying consecutively sequence of the screening operators

$$
S_{F_{1,2}[0]} \cdot S_{F_{2,3}[0]} \cdots S_{F_{m-3, m-2}[0]} \cdot S_{F_{m-2, m-1}[0]}
$$

to the both sides of the equality (4.45) and using the fact that the zero modes in these screening operators commute with the current $F_{m, m+1}^{+}(s)$ we get

$$
\begin{aligned}
&(s-t) F_{m, m+1}^{+}(s) P\left(F_{1, m}(t)\right)+s P\left(F_{1, m+1}(s)\right) \\
&=\left(q s-q^{-1} t\right) P\left(F_{1, m}(t)\right) F_{m, m+1}^{+}(s)+t\left(q^{-1}-q\right) P\left(F_{1, m}(t)\right) F_{m, m+1}^{+}(t) \\
&+t P\left(F_{1, m+1}(t)\right) .
\end{aligned}
$$

Comparing (4.43) and (4.46) proves the assertion of the Proposition 4.2.

\subsection{Calculation of the projections}

The recursion relation for the projection $P\left(F_{N-1, N}\left(t^{N-1}\right) \cdots F_{1,2}\left(t^{1}\right)\right)$ is given by the following

\section{Proposition 4.3.}

$$
\begin{aligned}
& P\left(F_{N-1, N}\left(t^{N-1}\right) \cdots F_{1,2}\left(t^{1}\right)\right) \\
& \quad=\sum_{m=2}^{N} P\left(F_{N-1, N}\left(t^{N-1}\right) \cdots F_{m, m+1}\left(t^{m}\right)\right) P\left(F_{1, m}\left(t^{m-1}\right)\right) \prod_{j=1}^{m-2} \frac{t^{j+1}}{t^{j+1}-t^{j}} .
\end{aligned}
$$

Proof of this proposition is based on the properties of the projections and the relation (4.37) which defines the composed currents. This relation can be written in the analytical form

$$
F_{s+1, j}(z) F_{i, s+1}(w)=\frac{q^{-1}-q z / w}{1-z / w} F_{i, s+1}(w) F_{s+1, j}(z)+F_{i, j}(z) \delta(z / w) .
$$

Any current $F_{i, j}(z)$ can be decomposed into difference of half-currents $F_{i, j}^{+}(z)-$ $F_{i, j}^{-}(z)$, where $F_{i, j}^{+}(z)=\sum_{n>0} F_{i, j}[n] z^{-n}$ and $F_{i, j}^{-}(z)=-\sum_{n \leq 0} F_{i, j}[n] z^{-n}$. Using this definition we may obtain from (4.48) the rule how to move negative halfcurrents through the total currents:

$$
\begin{aligned}
F_{s+1, j}(z) F_{i, s+1}^{-}(w) & =\frac{q z-q^{-1} w}{z-w} F_{i, s+1}^{-}(w) F_{s+1, j}(z) \\
& +\frac{\left(q^{-1}-q\right) z}{z-w} F_{i, s+1}^{-}(z) F_{s+1, j}(z)+\frac{z}{z-w} F_{i, j}(z) .
\end{aligned}
$$


We start to calculate left-hand side of (4.47) by decomposing current $F_{1,2}(z)=$ $F_{1,2}^{+}(z)-F_{1,2}^{-}(z)$ and using properties of the projections that

$$
P\left(F_{1} P\left(F_{2}\right)\right)=P\left(F_{1}\right) P\left(F_{2}\right) \quad \text { and } \quad P\left(\left(F_{1}-P\left(F_{1}\right)\right) F_{2}\right)=0,
$$

for any $F_{1}, F_{2} \in U_{f}$. We move negative half-current to the left using commutation relations (4.49). Since current $F_{1,2}\left(t^{1}\right)$ has non-trivial commutation relation only with the current $F_{2,3}\left(t^{2}\right)$ we obtain after this step two terms

$$
\begin{aligned}
P\left(F_{N-1, N}\left(t^{N-1}\right) \cdots\right. & \left.F_{2,3}\left(t^{2}\right)\right) \cdot P\left(F_{1,2}\left(t^{1}\right)\right) \\
& +P\left(F_{N-1, N}\left(t^{N-1}\right) \cdots F_{3,4}\left(t^{3}\right) F_{1,3}\left(t^{2}\right)\right) \frac{t^{2}}{t^{2}-t^{1}} .
\end{aligned}
$$

To calculate projection in the second line of (4.50) we will use a decomposition of the composed current $F_{1,3}\left(t^{2}\right)$ which follows from (4.39)

$$
F_{1,3}\left(t^{2}\right)=P\left(F_{1,3}\left(t^{2}\right)\right)+\left(q^{-1}-q\right)\left(F_{1,2}^{-}\left(t^{2}\right) F_{2,3}\left(t^{2}\right)\right)^{+}-F_{1,3}^{-}\left(t^{2}\right) .
$$

Here $(A(t))^{+}$means a projection of the current $A(t)$ onto half-current which contains the negative powers of the parameter $t^{2}$. Using again the commutation relation (4.49) and properties of the projections we obtain instead of (4.50)

$$
\begin{aligned}
& P\left(F_{N-1, N}\left(t^{N-1}\right) \cdots F_{2,3}\left(t^{2}\right)\right) \cdot P\left(F_{1,2}\left(t^{1}\right)\right) \\
& +P\left(F_{N-1, N}\left(t^{N-1}\right) \cdots F_{3,4}\left(t^{3}\right)\right) P\left(F_{1,3}\left(t^{2}\right)\right) \frac{t^{2}}{t^{2}-t^{1}} \\
& +P\left(F_{N-1, N}\left(t^{N-1}\right) \cdots F_{4,5}\left(t^{4}\right) F_{1,4}\left(t^{3}\right)\right) \frac{t^{2}}{t^{2}-t^{1}} \frac{t^{3}}{t^{3}-t^{2}} .
\end{aligned}
$$

Continuing further and using decomposition formulas similar to (4.51) we conclude the proof of the Proposition 4.3.

The statement of Theorem 1 follows now from the observation that multiplying the recursion relation (4.47) from the right by the product $\prod_{n=1}^{N-1} k_{n+1}\left(t^{n}\right)$ and acting onto weight singular vector $v$ we obtain

$$
\begin{aligned}
& P\left(F_{N-1, N}\left(t^{N-1}\right) \cdots F_{1,2}\left(t^{1}\right)\right) \prod_{n=1}^{N-1} k_{n+1}\left(t^{n}\right) v \\
& \quad=\sum_{m=2}^{N} P\left(F_{N-1, N}\left(t^{N-1}\right) \cdots F_{m, m+1}\left(t^{m}\right)\right) \prod_{n=m+1}^{N-1} k_{n+1}\left(t^{n}\right) \\
& \quad \times P\left(F_{1, m}\left(t^{m-1}\right)\right) k_{m}\left(t^{m-1}\right) \prod_{j=1}^{m-2}\left(\frac{t^{j+1}}{t^{j+1}-t^{j}} k_{j+1}\left(t^{j}\right)\right) v .
\end{aligned}
$$

It is obvious that this recursion relation coincides with (2.18) for the vector $\mathbb{B}_{[N-1]}\left(t^{1}, \ldots, t^{N-1}\right) v$. 


\section{References}

[EKhP] B. Enriquez, S. Khoroshkin, S. Pakuliak, Weight functions and Drinfeld currents, Preprint ITEP-TH-40/05.

[ER] B. Enriquez, V. Rubtsov, Quasi-Hopf algebras associated with $\mathfrak{s l}_{2}$ and complex curves, Israel J. Math 112 (1999), 61-108.

[DF] J. Ding, I.B. Frenkel, Isomorphism of two realizations of quantum affine algebra $U_{q}\left(\widehat{\mathfrak{g l}}_{N}\right)$, Comm. Math. Phys. 156 (1993), 277-300.

[DKh] J. Ding, S. Khoroshkin, Weyl group extension of quantized current algebras, Transformation Groups 5 (2000), 35-59.

[DKhP] J. Ding, S. Khoroshkin, S. Pakuliak, Integral presentations for the universal Rmatrix Lett. Math. Phys. 53 no. 2 (2000), 121-141; Factorization of the universal $R$-matrix for $U_{q}\left(\widehat{s l}_{2}\right)$ Theor. and Math. Phys. 124:2 (2000), 1007-1036.

[D] V. Drinfeld, New realization of Yangians and quantum affine algebras Sov. Math. Dokl. 36 (1988) 212-216.

$[\mathrm{KhP}]$ S. Khoroshkin, S. Pakuliak, Weight function for the quantum affine algebra $U_{q}\left(\widehat{\mathfrak{s l}}_{3}\right)$ Theor. and Math. Phys., 145 (1) (2005), 1373-1399.

[KhT] S. Khoroshkin, V. Tolstoy, Twisting of quantum (super)algebras. Connection of Drinfeld's and Cartan-Weyl realizations for quantum affine algebras, MPI Preprint MPI/94-23, hep-th/9404036.

[KR] P. Kulish, N. Reshetikhin, Diagonalization of $G L(N)$ invariant transfer matrices and quantum $N$-wave system (Lee model), J. Phys. A: Math. Gen. 16 (1983), L591-L596.

[RS] N. Reshetikhin, M. Semenov-Tian-Shansky, Central extentions of quantum current groups, Lett. Math. Phys. 19 (1990), 133-142.

[TV1] V. Tarasov, A. Varchenko, Jackson integrals for the solutions to KnizhnikZamolodchikov equation, Algebra and Analysis 2 (1995) no. 2, 275-313.

[TV2] V. Tarasov, A. Varchenko, Geometry of q-hypergeometric functions, quantum affine algebras and elliptic quantum groups, Astérisque 246 (1997), 1-135.

Stanislav Pakuliak

Laboratory of Theoretical Physics

Joint Institute of Nuclear Research

141980 Dubna

Moscow region

Russia

e-mail: pakuliak@theor.jinr.ru

Communicated by Vincent Rivasseau

Submitted: January 21, 2006

Accepted: March 13, 2006 